\title{
COEXTENSIONS OF PSEUDO-INVERSE SEMIGROUPS BY RECTANGULAR BANDS
}

\author{
JOHN MEAKIN and K. S. S. NAMBOORIPAD
}

(Received 31 October 1979)

Communicated by T. E. Hall

\begin{abstract}
We say that a regulär semigroup $S$ is a coextension of a (regular) semigroup $T$ by rectangular bands if there is a homomorphism $\varphi: S \rightarrow T$ from $S$ onto $T$ such that, for each $e=e^{2} \in S, e\left(\varphi=\varphi^{-1}\right)$ is a rectangular band. Regular semigroups which are coextensions of pseudo-inverse semigroups by rectangular bands may be characterized as those regular semigroups $S$ with the property that, for each $e=e^{2} \in S$, $\left(\omega^{r}\left(e^{\prime}\right)=\left\{f=f^{2} \in S: e f=f\right\}\right.$ and $\omega^{\prime}(e)=\left\{f=f^{2} \in S: f e=f\right\}$ are bands : this paper is concerned with a study of such semigroups.
\end{abstract}

1980 Mathematics subject classitication (Amer. Math. Soc.) : 20 M 10.

If $T$ is a regular semigroup we call a pair $(S, \varphi)$ consisting of a regular semigroup $S$ and a homomorphism $\varphi$ from $S$ onto $T$ a coextension of $T:(S, \varphi)$ is called a coextension of Thy rectangular bands if $e\left(\varphi \circ \varphi^{-1}\right)$ is a rectangular subband of $S$ for each idempotent $e$ of $S$. A general theory of coextensions of regular semigroups by rectangular bands has been developed by Meakin and Nambooripad (1979a, b). In the present paper we are concerned with coextensions of pseudo-inverse semigroups (see Nambooripad (19;8)) by rectangular bands : considerable simplifications in the general theory of Meakin and Nambooripad $(1979 a, b)$ occur in this case.

\section{Locally orthodox semigroups}

We assume familiarity with the general notation and basic results of semigroup theory as presented in the books of Clifford and Preston (1961) and Howie (1976).

The first author's research was supported partially by a grant form the U.S. National Science Foundation and was done while he was a Visiting Professor in the Department of Mathematics, University of Kerala.

The second author gratefully acknowledges financial assistance from the Indian University Grants Commission through Kerala University towards completing the research project. 
The set of idempotents of a subset $T$ of a regular semigroup $S$ will be denoted by $E(T)$, the set of inverses of an element $x \in S$ will be denoted by $V(x)$, and for $x \in S$ we denote by $\lambda_{x}\left[\rho_{x}\right]$ the left translation [right translation] by $x$. We also assume familiarity with the ideas, notation and results of Nambooripad's paper (1979) on the structure of regular semigroups and with Nambooripad's paper (1978) on pseudo-inverse semigroups. In particular, we recall the following notation from Nambooripad (1979). If $E$ is a biordered set and $e, f \in E$, we write $M(e, f)=\omega^{l}(e) \cap \omega^{r}(f)$. For $g, h \in M(e, f)$ we write $g \prec h$ if $e g \omega^{r} e h$ and $g f \omega^{l} h f:$ we write $g \equiv h$ if $g \prec h$ and $h \prec g$. Thus

$$
\begin{aligned}
S(e, f) & =\{h \in M(e, f): g \prec h \text { for all } g \in M(e, f)\} \\
& =\{h \in M(e, f): h \equiv g \text { for some } g \in S(e, f)\} .
\end{aligned}
$$

We also remark that we refer to the biordered set of idempotents of a pseudo-inverse semigroup as a pseudo-semilattice in this paper (it was called a partially associative pseudo-semilattice in Nambooripad (1978) : a pseudo-semilattice in our sense is also a pseudo-semilattice in the sense of Schein (1972), but not conversely). Thus a pseudo-semilattice is a biordered set $E$ in which $\omega(e)$ is a semilattice for each $e \in E$. Finally, we assume familiarity with the authors' papers (1979a) and (1979b) on coextensions of regular semigroups by rectangular bands.

We say that a regular semigroup $S$ is locally orthodox if, for every $f \in E(S), \omega^{r}(f)$ and $\omega^{l}(f)$ are subbands of $S$. If $S$ is a pseudo-inverse semigroup then from Nambooripad (1978) we know that for each $f \in E(S), \omega^{r}(f)$ is a right normal band and $\omega^{l}(f)$ is a left normal band: thus any pseudo-inverse semigroup is locally orthodox. It is clear that any orthodox semigroup is locally orthodox: other examples include

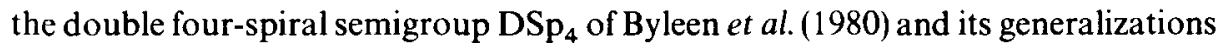
(see Byleen (1977)), which are neither orthodox nor pseudo-inverse.

We obtain some alternative characterizations of locally orthodox semigroups. First we prove the following proposition.

Proposition 1.1. Let $S$ be a regular semigroup and e, $f \in E(S)$ with e $\mathscr{L} f$. Assume that $\theta_{f}=\lambda_{f} \mid \omega^{r}(e)$ is an $\mathscr{L}$-class preserving mapping of $\omega^{r}(e)$ into $\omega^{r}(f)$. Then the following conditions are equivalent.

(a) $\theta_{e}=\lambda_{e} \mid \omega^{r}(f)$ is an $\mathscr{L}$-class preserving mapping of $\omega^{r}(f)$ into $\omega^{r}(e)$.

(b) $\theta_{f}$ is a bijection that preserves and reflects $\omega^{r}$ and $\omega^{l}$.

(c) For all $g \in E(S)$ and $h \in \omega^{r}(e), \theta_{f}(S(g, h))=S\left(g, \theta_{f} h\right)$.

When $\theta_{f}$ satisfies any one of these conditions, the $V(h)=V\left(\theta_{f} h\right)$ for all $h \in \omega^{r}(e)$.

Proof. (a) implies (b). If $\theta_{e}$ is a mapping from $\omega^{r}(f)$ to $\omega^{r}(e)$ then $\theta_{e}$ is clearly the inverse of $\theta_{f}$ and so $\theta_{f}$ is a bijection. Since $\theta_{f}$ is $\mathscr{L}$-class preserving, it is also $\omega^{l}$ preserving. To prove that $\theta_{f}$ preserves $\omega^{r}$, consider $g, h \in \omega^{r}(e)$ with $g \omega^{r} h$. Then 
ge $\omega^{r} h e, g e, h e \in \omega(e) \subseteq \omega^{l}(f)$. Hence $f(g e) \omega^{r} f(h e)$. Now $f g \in \omega^{r}(f)$ and $(f g)(f g e)=f g e$,

$$
(f g e)(f g)=(f g)(e f) g=f g e g=f g .
$$

Hence $f g \mathscr{R} f g e$. Similarly $f h \mathscr{R} f h e$. Hence $f g \omega^{r} f h$. Similarly $\theta_{e}$ also preserves $\omega^{l}$ and $\omega^{r}$; that is, $\theta_{f}$ reflects $\omega^{l}$ and $\omega^{r}$.

(b) implies (c). Suppose that $g \in E(S)$ and $h \in \omega^{r}(e)$. Choose $k \in S(g, h)$ and $k^{\prime} \in S\left(g, \theta_{f} h\right)$. Since $\theta_{f}$ preserves and reflects $\omega^{r}$ and $\omega^{l}$ there is a unique $k_{1} \in M(g, h)$ such that $f k_{1}=k^{\prime}$. Since $k \in S(g, h)$ we have $k_{1} \prec k$ and so by Nambooripad (1979), Proposition 2.10, there exists $k_{2} \in M(g, h)$ such that $k_{1} \equiv k_{2} \omega k$. Then $f k_{2} \omega f k$ and since $f k \in M(g, f h), f k \prec k^{\prime}=f k_{1}$. Hence $f k_{2} \prec k^{\prime}$. But $f k_{2}$ is in an $E$-square with $k^{\prime}$ and so by Meakin and Nambooripad (1979a), Corollary 1.9, $f k_{2} \equiv k^{\prime}$. Hence $f k \prec k^{\prime} \equiv f k_{2}$. This together with the relation $f k_{2} \omega f k$ implies that $f k_{2}=f k$ and so $f k \in S(g, f h)$. Again since $\theta_{f}$ is a bijection, we have $k_{2}=k$. Since $k_{1} \equiv k_{2}=k$, we again have $k_{1} \in S(g, h)$. Therefore, $f S(g, h)=S(g, f h)$.

(c) implies (a). Let $k^{\prime} \in \omega^{r}(f)$. We shall show that $e k^{\prime}$ is an idempotent. Now $k_{1}=e k^{\prime} f \in \omega^{\prime}(e)$ and so, by (c),

$$
f S\left(k^{\prime}, k_{1}\right)=S\left(k^{\prime}, f k_{1}\right)=S\left(k^{\prime}, k^{\prime} f\right)=\left\{k^{\prime}\right\} .
$$

Hence if $k \in S\left(k^{\prime}, k_{1}\right)$, then $f k=k^{\prime}$ so $k \mathscr{L} k^{\prime}$. Therefore $k^{\prime} \in L_{k} \cap R_{k^{\prime} f}$ and so $k f=k k^{\prime} f \in R_{k} \cap L_{k^{\prime} f}$. Therefore $k \mathscr{R} k f \mathscr{L} e k^{\prime} f=k_{1}$. But since $k \in S\left(k^{\prime}, k_{1}\right), k f \in \omega^{r}\left(k_{1}\right)$. Hence $k f=k_{1}$. Therefore $k \in R_{k_{1}} \cap L_{k^{\prime}}$. But $\quad k_{1} k^{\prime}=e k^{\prime} \in R_{k_{1}} \cap L_{\mathbf{k}^{\prime}}$ and $f\left(e k^{\prime}\right)=k^{\prime}=f k$. Therefore $e k^{\prime}=k \in \omega^{r}(f)$. This proves that $\theta_{e}: \omega^{r}(f) \rightarrow \omega^{r}(e)$ is an $\mathscr{L}$-class preserving map of $\omega^{r}(f)$ into $\omega^{r}(e)$ and hence (a) holds.

If $\theta_{f}$ satisfies these conditions, then for any $k \in \omega^{r}(e), \theta_{f} \mid E\left(R_{k}\right)$ is an $\mathscr{L}$-class preserving bijection onto $E\left(R_{f k}\right)$ and hence the final assertion follows from Lemma 2.1 of Meakin and Nambooripad (1979a).

We recall from Meakin and Nambooripad (1979a) that if $S$ is any regular semigroup then

$$
\pi(S)=\{(x, y) \in S \times S: V(x)=V(y)\}
$$

and that we denote the maximum congruence contained in $\pi(S)$ by $\rho(S)$. Recall also from Meakin and Nambooripad (1979a), Proposition 2.3, that for any congruence $v$ on $S, S$ is a coextension of $S / v$ by rectangular bands if and only if $v \subseteq \rho(S)$. In order to characterize $\rho(S)$ we introduced in Meakin and Nambooripad (1979a) the following concept of similarity between principal right [left] ideals.

DEFINITION 1.2. If $S$ is a regular semigroup and $x, y \in S$ we say that the right ideals $x S$ and $y S$ are similar, written $x S \approx y S$, if there exist $e \in E\left(R_{x}\right)$ and $f \in E\left(R_{y}\right)$ such that $e \mathscr{L} f$ and $\theta_{f}=\left.\lambda_{f}\right|_{E(x S)}$ is an $\mathscr{L}$-class-preserving bijection of $\omega^{r}(e)$ onto $\omega^{r}(f)$ satisfying the following conditions. 
(1) for all $g \in E(S)$ and $k \in S(g, e), \theta_{f}(S(g, e))=S(g, f)$ and $\left(k e,\left(\theta_{f} k\right) f\right) \in \pi(S)$.

(2) for all $u \in S, u^{\prime} \in V(u)$ and $k \in S\left(u^{\prime} u, e\right),\left(u k u^{\prime}, u \theta_{f} k u^{\prime}\right) \in \pi(S)$.

Similarity between principal left ideals is defined dually. The characterization of $\rho(S)$ provided in Meakin and Nambooripad (1979a) is the following.

THEOREM 1.3. (Meakin and Nambooripad (1979a), Theorem 2.9.) Let $S$ be a regular semigroup. Then $(x, y) \in \rho(S)$ if and only if $x S \approx y S, S x \approx S y$ and $y=$ exf for some $e \in E\left(R_{y}\right)$ and $f \in E\left(L_{y}\right)$.

If $S$ is locally orthodox we are able to provide much more definitive information about the congruence $\rho(S)$ than we can provide in the general case. This is provided by the following characterization of similarity between principal right (left) ideals.

THEOREM 1.4. Suppose that $x S$ and $y S$ are two right ideals of a locally orthodox semigroup $S$ and let $e \in E\left(R_{x}\right), f \in E\left(R_{y}\right)$ with $e \mathscr{L} f$. Then $x S \approx y S$ if and only if $\theta_{f}=\lambda_{f} \mid \omega^{r}(e): \omega^{r}(e) \rightarrow \omega^{r}(f)$ is an $\mathscr{L}$-class preserving bijection that preserves and reflects $\omega^{r}$ and $\omega^{l}$.

Proof. If $x S \approx y S$ then it follows from Proposition 2.8 of Meakin and Nambooripad (1979a) that $\theta_{f}: \omega^{r}(e) \rightarrow \omega^{r}(f)$ is an $\mathscr{L}$-class preserving bijection that preseves and reflects $\omega^{r}$ and $\omega^{l}$. Conversely if $\theta_{f}$ has this property we shall show that $\theta_{f}$ satisfies conditions (1) and (2) of the definition of similarity. By Proposition 1.1, $f S(g, e)=S(g, f)$ and $V(k)=V(f k)$ for all $g \in E(S)$ and $k \in S(g, e)$. Since $f k e=\theta_{f}(k e)$, $f k f=\left(\theta_{f} k\right) f, f k e$ and $f k f$ are idempotents in the band $\omega^{l}(f)$ such that $f k e \mathscr{R} f k f$ and so by Lemma 2.1 of Meakin and Nambooripad (1979a), $V(f k e)=V(f k f)$. If $c \in S$, $c^{\prime} \in V(c)$ and $h \in S\left(c^{\prime} c, e\right)$ then $c h c^{\prime}$ and $c\left(\theta_{f} h\right) c^{\prime}$ are $\mathscr{L}$-equivalent idempotents in $\omega^{r}\left(c c^{\prime}\right)$ and so, as before, we conclude by Lemma 2.1 of Meakin and Nambooripad (1979a) that $V\left(c h c^{\prime}\right)=V\left(c\left(\theta_{f} h\right) c^{\prime}\right)$. Hence $\theta_{f}$ satisfies the conditions (1) and (2) and so $x S=e S \approx f S=y S$.

THEOREM 1.5. The following statements are equivalent for a regular semigroup $S$.

(a) $S$ is locally orthodox.

(b) $S / \rho(S)$ is a pseudo-inverse semigroup.

(c) For all $e, f \in E(S)$ and $g, h \in M(e, f)$, if $V(g) \cap V(h) \neq \emptyset$ then $V(g)=V(h)$.

(d) $S$ is a coextension of a pseudo-inverse semigroup by rectangular bands.

Proof. (a) implies (b). Let $S / \rho(S)=\bar{S}$ and $E(\bar{S})=E^{\prime}$. We shall show that $E^{\prime}$ is a pseudo-semilattice. Consider $e^{\prime} \in E^{\prime}$ and $g^{\prime}, h^{\prime} \in \omega\left(e^{\prime}\right)$ with $g^{\prime} \mathscr{L} h^{\prime}$. If $e \in E=E(S)$ with $e \theta=e^{\prime}$ (where $\theta=E\left(\rho^{\natural}\right)$ ), then we claim that we can find $g, h \in \omega(e)$ such that $g \mathscr{L} h$ and $g \theta=g^{\prime}, h \theta=h^{\prime}$. For by Nambooripad (1979a), Proposition 2.14, there exist $g, h \in \omega(e)$ such that $g \theta=g^{\prime}, h \theta=h^{\prime}$. By Lemma 2.4 of Meakin and Nambooripad 
(1979a), $E_{y^{\prime}} \cup E_{h^{\prime}}$ (where $E_{k^{\prime}}=k^{\prime} \theta^{-1}$ ) is a rectangular band. Hence $g \mathscr{L} h g$ and $h g \in E_{h^{\prime}} \cap \omega(e)$. This proves our claim. We shall now show that $(g, h) \in \rho(S)$ (where $g \mathscr{L} h, g \theta=g^{\prime}$ and $\left.h \theta=h^{\prime}\right)$. Since $\omega^{r}(e)$ is a band,

$$
\theta_{h}=i_{h} \mid \omega^{r}(g): \omega^{r}(g) \rightarrow \omega^{r}(h) \text { and } \theta_{g}: \omega^{r}(h) \rightarrow \omega^{r}(g)
$$

are $\mathscr{L}$-class preserving maps. Therefore by Proposition $1.1, h S(f, g)=S(f, h)$ and $V(k g)=V(h k g)$ for all $f \in E(S)$ and $k \in S(f, g)$. Also $h k g, h k h \in \omega^{l}(g)$ and $h k g \mathscr{R} h k h$.

Since $\omega^{l}(g)$ is a band, we have by Lemma 2.1 of Meakin and Nambooripad (1979a), that $V(h k g)=V(h k h)$. Therefore $V(k g)=V(h k h)$ and this proves that condition (1) above is satisfied. To prove that condition (2) is satisfied, consider $c \in S, c^{\prime} \in V(c)$ and $k \in S\left(c^{\prime} c, g\right)$. Then $c k c^{\prime}$ and $c h k c^{\prime}=c\left(\theta_{h} k\right) c^{\prime}$ are $\mathscr{L}$-equivalent idempotents in the band $\omega^{\prime}\left(c c^{\prime}\right)$ and so, as before, we conclude that $V\left(c k c^{\prime}\right)=V\left(c\left(\theta_{h} k\right) c^{\prime}\right)$. Therefore by Theorem 1.3, $(g, h) \in \rho(S)$. Hence $g^{\prime}=g \theta=h \theta=h^{\prime}$. If $g^{\prime}, h^{\prime} \in \omega\left(e^{\prime}\right)$ and $g^{\prime} \mathscr{R} h^{\prime}$ we can similarly show that $g^{\prime}=h^{\prime}$. Therefore the relations $\omega^{r}$ and $\omega^{l}$ coincide with $\omega$ on $\omega\left(e^{\prime}\right)$ and so $\omega\left(e^{\prime}\right)$ is a semilattice. This proves that $E^{\prime}$ is a pseudo-semilattice.

(b) implies (c). Let $y, h \in M(e, f)$ and $x \in V(g) \cap V(h)$. If $\theta=E\left(\rho(S)^{\natural}\right)$, then

$$
g \theta \mathscr{R}(y x) \theta \mathscr{L}(h x) \theta \mathscr{R} h \theta \text { in } E^{\prime}=E(S / \rho(S)) .
$$

Since $(g x) \theta,(h x) \theta \in \omega^{r}(f \theta)$ and $\omega^{r}(f \theta)$ is a right normal band, $(g x) \theta=(h x) \theta$. Then $g \theta, h \theta \in \omega^{l}(e \theta)$ and $g \theta \mathscr{R} h \theta$. Then again since $\omega^{l}(e \theta)$ is a left normal band, $g \theta=h \theta$. Hence $(g, h) \in \rho(S)$ and so $V(g)=V(h)$.

(c) implies (a). Let $e \in E(S)$ and $f, g \in \omega^{r}(e)$. If $h \in S(f, g)$, then $h \mathscr{L} f h$ and $h, f h \in \omega^{r}(e) \cap \omega^{l}(f)=M(e, f)$. Also $h \in V(h) \cap V(f h)$ and so $V(h)=V(f h)$ by $(c)$. Now $f \in V(h)$ and so $e f \in V(f h)$ and $e f \mathscr{R} f h$. Since $f h$ is an idempotent, it follows that $e f$ is also an idempotent. Therefore $\omega^{r}(e)$ is a band. Dually $\omega^{l}(e)$ is also a subband of $S$. This proves that $S$ is locally orthodox. It is clear that (b) and (d) are equivalent, so this completes the proof.

Recall that the $E(S)$ of idempotents of a regular semigroup $S$ relative to the multiplication $e \cdot f=e f$ if $e f \in E(S), e \cdot f$ is undefined if $e f \notin E(S)$ becomes a regular partial band and that the structure of regular partial bands is determined in Nambooripad (1979). We define a local band to be a regular partial band $E$ in which $\omega^{r}(e)$ and $\omega^{l}(e)$ are bands for each $e \in E$.

From the definition of a local band we obviously have the following proposition.

PROPOSITION 1.6. A regular semigroup $S$ is locally orthodox if and only if its regular partiai band is a local band.

We recall from Meakin and Nambooripad (1979a) that if $B_{1}$ and $B_{2}$ are regular partial bands, then we say that $\left(B_{1}, \theta\right)$ is a strong coextension of $B_{2}$ if $\theta$ is a (regular partial band) homomorphism from $B_{1}$ onto $B_{2}$ and the product $e f$ is defined in $B_{1}$ if 
and only if the product $(e \theta)(f \theta)$ is defined in $B_{2}$. (In this case we also call $B_{1}$ a strong coextension of $B_{2}$.) Strong coextensions of a regular partial band $B$ by rectangular bands were constructed in Meakin and Nambooripad (1979a). We have the following result describing the structure of local bands.

THEOREM 1.7. A regular partial band $B$ is a local band if and only if $B$ is a strong coextension by rectangular bands of a regular partial band whose biordered set is a pseudo-semilattice.

Proof. Let $S$ be a regular semigroup whose regular partial band is $B$. If $B$ is a local band it follows from Proposition 1.6 that $S$ is locally othodox and so by Theorem $1.5(\mathrm{~b}), B$ is a strong coextension by rectangular bands of a regular partial band whose biordered set is a pseudo-semilattice. Conversely, assume that $(B, \theta)$ is astrong coextension by rectangular bands of a regular partial band $B^{\prime}$ whose biordered set $E\left(B^{\prime}\right)$ is a pseudo-semilattice. Then for every $e \in B, \omega^{\prime}(e) \theta=\omega^{\prime}(e \theta)$ and $\omega^{l}(e) \theta=\omega^{l}(e \theta)$. Since $E\left(B^{\prime}\right)$ is a pseudo-semilattice, $\omega^{r}(e \theta)$ and $\omega^{l}(e \theta)$ are subbands of $B^{\prime}$ and since $\theta$ is strong, $\omega^{r}(e)$ and $\omega^{l}(e)$ are subbands of $B$. Thus $B$ is a local band.

We recall from Meakin and Nambooripad (1979b) that if $\theta$ is a bimorphism from the biordered set $E$ onto the biordered set $E^{\prime}$ then we denote by $\mathscr{T}_{\theta}$ the full inductive subgroupoid of $T^{*}(E)$ consisting of the $\omega$-isomorphisms $\alpha \in T^{*}(E)$ which are compatible with $\theta: \mathscr{T}_{\theta}$ is the maximum subgroupoid of $T^{*}(E)$ to which $\theta$ extends as an inductive functor, and the extension is unique (Meakin and Nambooripad (1979b), Proposition 1.2). Again, as in Meakin and Nambooripad (1979b), we denote the associated semigroup $S\left(\mathscr{T}_{\theta}\right)=\mathscr{T}_{\theta} / \rho$ by $T_{\theta}$.

If $B$ is a band and if $\theta$ is the homomorphism of $B$ onto its structure semilattice, then we know that $T_{\theta}=T(B)$. This holds for local bands as well. This is clear from the following result.

THEOREM 1.8. Let $(E, \theta)$ be a coextension of a biordered set $E^{\prime}$ by rectangular biordered sets. If $E^{\prime}$ is a pseudo-semilattice, then $\bar{\theta}$ is a homomorphism of $T(E)$ into $T\left(E^{\prime}\right)$; that is, $T_{\theta}=T(E)$.

ProOf. We shall show that $\mathscr{T}_{\theta}=T^{*}(E)$. Let $\alpha \in T^{*}(E)$. Define $\alpha^{\prime}$ by :

$$
(e \theta) \alpha^{\prime}=(e \alpha) \theta
$$

for all $e \in \omega\left(e_{\alpha}\right)$. If $e \theta=f \theta, e, f \in \omega\left(e_{\alpha}\right)$, then there exists $g \in \omega\left(e_{\alpha}\right)$ such that $e \mathscr{L} g \mathscr{R} f$ and $e \theta=g \theta=f \theta$ since $(e \theta) \theta^{-1}$ is rectangular. Then $e \alpha \mathscr{L} g \alpha \mathscr{R} f \alpha, e \alpha, g \alpha, f \alpha \in \omega\left(f_{\alpha}\right)$. Therefore

$$
(e \alpha) \theta,(g \alpha) \theta,(f \alpha) \theta \in \omega\left(f_{\alpha} \theta\right) \text { and }(e \alpha) \theta \mathscr{L}(g \alpha) \theta \mathscr{R}(f \alpha) \theta
$$


Since $E^{\prime}$ is a pseudo-semilattice, $\omega\left(\mathrm{f}_{\alpha} \theta\right)$ is a semilattice. Therefore $(e \alpha) \theta=(g \alpha) \theta=(f \alpha) \theta$. Thus $\alpha^{\prime}$ is single-valued. In a similar way we see that $\left(\alpha^{-1}\right)^{\prime}$ is also single-valued. Also for $e \in \omega\left(e_{\alpha}\right), \quad\left((e \theta) \alpha^{\prime}\right)\left(\alpha^{-1}\right)^{\prime}=\left(e \alpha \alpha^{-1}\right) \theta=e \theta$. Since $\omega\left(e_{\alpha}\right) \theta=\omega\left(e_{\alpha} \theta\right)$ and $\omega\left(f_{\alpha}\right) \theta=\omega\left(f_{\alpha} \theta\right)$, it follows that $\alpha^{\prime}$ is a bijection of $\omega\left(e_{\alpha} \theta\right)$ onto $\omega\left(\mathrm{f}_{x} \theta\right)$. If $e, f \in \omega\left(e_{x}\right)$, and if the basic product $e f$ exists, then

$$
(e \theta f \theta) \alpha^{\prime}=((e f) \theta) \alpha^{\prime}=((e f) \alpha) \theta=(e \alpha) \theta(f \alpha) \theta=(e \theta) \alpha^{\prime}(f \theta) \alpha^{\prime} .
$$

Since $\theta$ reflects basic products, this proves that $\alpha^{\prime} \in T^{*}\left(E^{\prime}\right)$ and so $\theta^{*}(\alpha)=\alpha^{\prime}$. Hence $\mathscr{T}_{\theta}=T^{*}(E)$. It follows immediately that $T_{\theta}=S\left(\mathscr{T}_{\theta}\right)=S\left(T^{*}(E)\right)=T(E)$.

COROLLARY 1.9. Let $S$ be a regular semigroup and $v$ be a biorder congruence on $E(S)$ whose congruence classes are rectangular biordered subsets, such that $E(S) / v$ is a pseudo-semilattice. Then $v$ extends to a congruence on $S$.

Proof. If $\theta=v^{\natural}$ then by Theorem $1.8, T_{\theta}=T(E)$, so $\operatorname{ker} \bar{\theta}$ is a congruence on $T(E)$ such that the biorder congruence on $E(T(E))=E$ induced by $\operatorname{ker} \bar{\theta}$ is $v$. Then

$$
\bar{v}=\left\{(x, y) \in S \times S:\left(x \bar{a}_{S}\right) \bar{\theta}=\left(y \bar{a}_{s}\right) \bar{\theta}\right\}
$$

is an extension of $v$. (Here $\bar{a}_{S}$ is the natural homomorphism from $S$ to $T(E)$-see Nambooripad (1979).)

Let $B$ be a band. A congruence $v$ on $B$ is called a strong pseudo-semilattice congruence on $B$ if $B / v$ is a regular partial band such that $E(B / v)$ is a pseudosemilattice and $(B, v)$ is a strong coextension of $B / v$ by rectangular bands.

COROLlary 1.10. Let $S$ be a locally orthodox semigroup and $v$ be a strong pseudosemilattice congruence on $B(S)$. Then $v$ has a unique extension $\bar{v}$ to a congruence on $S$ such that $\bar{v} \subseteq \rho(S)$.

Proof. We shall prove that $e v \subseteq e \rho(S)$ for all $e \in B(S)$. (Here $B(S)$ is the regular partial band of $S$.) Consider $f \in e v$ with $e \mathscr{L} f$. If $h \in \omega^{r}(e)$ then it is clear that $h \mathscr{L} f h$. Also $h v^{\natural} \omega^{r} e v^{\natural}=f v^{\natural}$ and so the product $f v^{\natural} h v^{\natural}$ exists in $B(S) / v$. Since $v$ is strong it follows that the product $f h$ exists in $B(S)$. Thus $\theta_{f}=\lambda_{f} \mid \omega^{r}(e)$ is an $\mathscr{L}$-class preserving map of $\omega^{r}(e)$ into $\omega^{r}(f)$. Similarly $\theta_{e}$ is also an $\mathscr{L}$-class preserving map of $\omega^{r}(f)$ into $\omega^{r}(e)$. Therefore, by Proposition 1.1 and Theorem 1.4, $(e, f) \in \rho(S)$. Dually if $e \mathscr{R} f$ we can show that $(e, f) \in \rho(S)$. Since $e v$ is a rectangular band, it follows that $e v \subseteq e \rho(S)$.

Now by Corollary 1.9, there is a congruence $\sigma$ on $S$ such that $\sigma \cap(B(S) \times B(S))=v$. Hence if $\bar{v}=\sigma \cap \rho(S)$, then $\bar{v}$ is a congruence on $S$ such that $\bar{v} \cap(B(S) \times B(S))=v$. Since $\bar{v} \subseteq \rho(S)$, it is also unique.

The foregoing result shows that on any local band $B$ there exists a maximum strong pseudo-semilattice congruence, namely the congruence $\rho(S) \cap(B(S) \times B(S))$ 
where $S$ is any locally orthodox semigroup with $B(S)=B$. We shall denote this by $\rho(B)$. We shall say that a local band $B$ is $\rho$-irreducible if $\rho(B)$ is the identity congruence on $B$. In this case, it is clear that $E(B)$ is a pseudo-semilattice.

COROLLARY 1.11. Every local band is a strong coextension of a $\rho$-irreducible local band.

COROLLARY 1.12. A locally orthodox semigroup $S$ is $\rho$-irreducible if and only if it is a pseudo-inverse semigroup whose local band is $\rho$-irreducible.

It is clear that, using the results of Meakin and Nambooripad (1979b), every locally orthodox semigroup can be constructed from $\rho$-irreducible pseudo-inverse semigroups and local bands. In view of Theorem 1.8, one can see that $T_{\theta}=T(E)$ and hence this construction is an exact generalization of Hall's construction of orthodox semigroups from bands and inverse semigroups (compare with Hall (1971), Theorem 1). We shall provide some additional information about $\rho$-irreducible pseudo-inverse semigroups in Section 2.

\section{Normal coextensions of pseudo-inverse semigroups}

We recall from Meakin and Nambooripad (1979a) that a coextension $(E, \theta)$ of a biordered set $F$ is called normal if it satisfies the condition :

(N) $\quad$ if $\beta \omega \alpha$ in $F$ and $e \theta=\alpha$, then there is a unique $f \in E$ such that $f \theta=\beta$ and $f \omega e$.

We saw in Meakin and Nambooripad (1979a) that a normal coextension of a biordered set $F$ must be a coextension of $F$ by rectangular biordered sets and we provided a construction of such coextensions. In the present section we study normal coextensions of pseudo-semilattices.

THEOREM 2.1. Let $E^{\prime}$ be a pseudo-semilattice and let $(E, \theta)$ be a coextension of $E^{\prime} b y^{\prime}$ rectangular biordered sets. Then $E$ is also a pseudo-semilattice if and only if the coextension is normal.

Proof. Suppose first that $E$ is a pseudo-semilattice. If $e^{\prime} \omega f^{\prime}$ in $E^{\prime}$ and if $f \in E_{f^{\prime}}=f^{\prime} \theta^{-1}$, then there exists $e \in E_{e^{\prime}}$ such that $e \omega f$. If $e_{1} \in E_{e^{\prime}}$ and $e_{1} \omega f$, then since $E_{e^{\prime}}$ is a rectangular biordered set, there exists $e_{2} \in E_{e^{\prime}}$ with $e \mathscr{L} e_{2} \mathscr{R} e_{1}$. Then $e_{2} \omega f$ : since $\omega(f)$ is a semilattice, $e=e_{2}=e_{1}$. Therefore $E_{e^{\prime}}$ contains a unique element $e$ such that $e \omega f$. Therefore the coextension $(E, \theta)$ is normal.

Conversely, assume that $(E, \theta)$ is a normal coextension of $E^{\prime}$. If $e_{1}, e_{2} \in W(f)(f \in E)$ with $e_{1} \mathscr{R} e_{2}$ then $e_{1} \theta \mathscr{R} e_{2} \theta$ and $e_{1} \theta, e_{2} \theta \in \omega(f \theta)$. Since $E^{\prime}$ is a pseudo-semilattice we 
have $e_{1} \theta=e_{2} \theta$. In view of the normality of the coextension $(E, \theta)$, this implies that $e_{1}=e_{2}$. If $e_{1} \mathscr{L} e_{2}$ we can dually show that $e_{1}=e_{2}$. Hence $\omega(f)$ is a semilattice. Therefore $E$ is a pseudo-semilattice.

REMark. The foregoing theorem shows that the class of pseudo-semilattices is closed relative to taking normal coextensions. Schein's result (Schein (1972)) that normal bands are pseudo-semilattices is an immediate consequence of this fact.

It follows from Theorem 2.1 that if $S$ is a pseudo-inverse semigroup and $v$ is a congruence on $S$ contained in $\rho(S)$, then $\left(S, v^{\natural}\right)$ is a normal coextension of $S / v$. We are able to obtain a further simplification of $\rho(S)$ in the case where $S$ is a pseudo-inverse semigroup. This is provided by Theorem 1.3 and the following result, which sharpens the characterization of similarity between principal left [right] ideals in this case.

THEOREM 2.2. Let $S$ be a pseudo-inverse semigroup, $x, y \in S$ and $e \in E\left(R_{x}\right), f \in E\left(R_{y}\right)$ withe $\mathscr{L} f$. Then $x S \approx y S$ if and only if there is an $\mathscr{L}$-class preserving isomorphism $\theta$ of $\omega^{r}(e)$ onto $\omega^{r}(f)$ such that for all $h \in \omega^{r}(e)$ the E-square

$$
\left(\begin{array}{cc}
h & h e \\
\theta(h) & \theta(h e)
\end{array}\right)
$$

is a $(2 \times 2)$ rectangular band.

Proor. If $x S=e S \approx f S=y S$ then by Theorem $1.4, \theta_{f}$ is a bijection that preserves and reflects $\omega^{r}$ and $\omega^{l}$. Then by Proposition 1.1, for all $g, h \in \omega^{r}(e)$. $\theta_{f}(S(g, h))=S\left(g, \theta_{f} h\right)$; that is, $\theta_{f}(g \wedge h)=g \wedge \theta_{f} h$. Since $\theta_{f} g \mathscr{L} g$ we have $\theta_{f} g \wedge \theta_{f} h=g \wedge \theta_{f} h$. Since $h g=g \wedge h$ we have

$$
\theta_{f}(h g)=\theta_{f}(h) \theta_{f}(g) \text {. }
$$

Hence $\theta_{f}$ is an isomorphism. The last condition follows from the equality $f h=(f h e) h$.

Conversely if $\theta: \omega^{r}(e) \rightarrow \omega^{r}(f)$ is an isomorphism, we must show that $\theta(h)=f h$ for all $h \in \omega^{r}(e)$. Since $\theta$ preserves the relations $\mathscr{R}$ and $\mathscr{L}$ on $\omega^{r}(e)$, it follows that $\theta(e) \mathscr{R} f$. Since $e \mathscr{L} \theta(e)$, we must have $\theta(e)=f$. If $h \in \omega^{r}(e)$ then $\theta(h e)$ and $f h$ are $\mathscr{L}$-equivalent idempotents in $\omega(f)$ and since $\omega(f)$ is a semilattice, we have $\theta($ he $)=f$ he. Now by the given condition

$$
\theta(h)=\theta(h e) h=(f h e) h=f h .
$$

Hence $\theta=\theta_{f}=i_{f} \mid \omega^{r}(e)$ and this completes the proof of the theorem.

We turn now to a consideration of $\rho$-irreducible pseudo-inverse semigroups : $\rho$ irreducibility of regular semigroups is not, in general, a biordered set property, but for locally orthodox and pseudo-inverse semigroups, Corollary 1.12 shows that it is 
a property of their regular partial bands. However, it is possible to characterize those pseudo-semilattices $E$ with the property that all pseudo-inverse semigroups $S$ with $E(S)=E$ are $\rho$-irreducible and those with the property that there exists some $\rho$ irreducible pseudo-inverse semigroup $S$ with $E(S)=E$.

We first introduce the following concept of equivalence of right [left] ideals of a pseudo-semilattice $E$. If $e, f \in E$ we say that $\omega^{r}(e)$ and $\omega^{r}(f)$ are equivalent if there exists an $\mathscr{L}$-class preserving isomorphism $\theta: \omega^{r}(e) \rightarrow \omega^{r}(f)$ such that for $g, h \in \omega^{r}(e)$ with $g h$ the following diagram commutes.

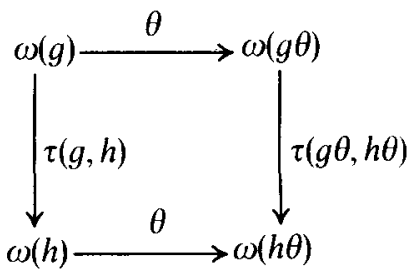

It may be noted that the equivalence defined above is a biordered set property. The reader may compare this with the concept of similarity between two right [left] ideals defined earlier. If $S$ and $S^{\prime}$ are regular semigroups with $E(S)=E\left(S^{\prime}\right)=E$, then for $e, f \in E$ it may happen that $e S \approx f S$, but $e S^{\prime}$ is not similar to $f S^{\prime}$. Theorem 1.4 shows that for locally orthodox semigroups, similarity is a regular partial band property. It may be noted that even for pseudo-inverse semigroups, the similarity of right [left] ideals is not a biordered set property. The next theorem clarifies the relationship between the two concepts of similarity and equivalence in the pseudoinverse case.

THEOREM 2.3. Let $S$ be a pseudo-inverse semigroup and e, $f \in E=E(S)$. If $(e, f) \in \rho(S)$ then the right $[$ left $]$ ideals $\omega^{r}(e)\left[\omega^{l}(e)\right]$ and $\omega^{r}(f)\left[\omega^{l}(f)\right]$ are equivalent. Conversely, if $\omega^{r}(e)$ and $\omega^{r}(f)$ are equivalent and $\omega^{l}(e)$ and $\omega^{l}(f)$ are equivalent, then $(e, f) \in \rho(T(E))$.

Proof. Suppose that $(e, f) \in \rho=\rho(S)$. Then $\theta_{f}=\lambda_{f} \mid \omega^{r}(e)$ is an $\mathscr{L}$-class preserving isomorphism of $\omega^{r}(e)$ onto $\omega^{r}(f)$ such that for all $h \in \omega^{r}(e)$ the $E$-square

$$
\left(\begin{array}{cc}
h & h e \\
\theta_{f}(h) & \theta_{f}(h e)
\end{array}\right)
$$

is a $2 \times 2$ rectangular band in $S$. So if $g \mathscr{R} h$, it follows that

$$
\left(\begin{array}{cc}
g & g e \\
\theta_{f}(g) & \theta_{f}(g e)
\end{array}\right) \text { and }\left(\begin{array}{cc}
h & h e \\
\theta_{f}(h) & \theta_{f}(h e)
\end{array}\right)
$$


are $\tau$-commutative $E$-squares. Since $\theta_{f} \mid \omega(e)=\tau(e, f)$ it follows that

$$
\left(\begin{array}{cc}
g e & h e \\
\theta_{f}(g e) & \theta_{f}(h e)
\end{array}\right)
$$

is $\tau$-commutative since it is singular. Hence

$$
\left(\begin{array}{cc}
g & h \\
\theta_{f}(g) & \theta_{f}(h)
\end{array}\right)
$$

is $\tau$-commutative. Thus $\omega^{r}(e)$ and $\omega^{r}(f)$ are equivalent. Similarly $\omega^{l}(e)$ and $\omega^{l}(f)$ are equivalent.

Conversely if $\omega^{r}(e)$ and $\omega^{r}(f)$ are equivalent and if $\theta_{1}: \omega^{r}(e) \rightarrow \omega^{r}(f)$ is the $\mathscr{L}$-class preserving isomorphism, then for all $h \in \omega^{r}(e)$,

$$
\left(\begin{array}{cc}
h & h e \\
\theta_{1}(h) & \theta_{1}(h e)
\end{array}\right)
$$

is $\tau$-commutative. By the definition of product in $T(E)$ this is equivalent to the requirement that

$$
\left(\begin{array}{cc}
h & h e \\
\theta_{1}(h) & \theta_{1}(h e)
\end{array}\right)
$$

is a rectangular band in $T(E)$. Hence by Theorem $2.2, e T(E) \approx f T(E)$. Dually, $T(E) e \approx T(E) f$. Let $\theta_{2}: \omega^{l}(e) \rightarrow \omega^{l}(f)$ be the $\mathscr{R}$-class preserving isomorphism whose existence is guaranteed by the defintion of equivalence of $\omega^{r}(e)$ with $\omega^{r}(f)$ and of $\omega^{t}(e)$ with $\omega^{l}(f)$. Clearly

$$
\left(\begin{array}{cc}
e & \theta_{2}(e) \\
\theta_{1}(e) & f
\end{array}\right)
$$

is an $E$-square which is $\tau$-commutative by definition of equivalence. Hence

$$
\left(\begin{array}{cc}
e & \theta_{2}(e) \\
\theta_{1}(e) & f
\end{array}\right)
$$

is a rectangular band in $T(E)$ and so $(e, f) \in \rho(S)$ by Theorem 1.3.

As an immediate corollary we have:

COROLLARY 2.4. Let E be a pseudo-semilattice. Then every pseudo-inverse semigroup with biordered set $E$ is $\rho$-irreducible if and only if no two distinct $\omega^{r}$-ideals or $\omega^{l}$-ideals are equivalent.

If $E$ is a pseudo-semilattice, by Corollary 3.3 of Meakin and Nambooripad (1979b), $T(E) / \rho(T(E))$ is isomorphic to $T\left(E^{\prime}\right)$ where $E^{\prime}=E / E(\rho(T(E))$. Since $T(E) / \rho(T(E))$ is $\rho$-irreducible, it follows that evey pseudo-inverse semigroup $S$ with 
$E(S)=E^{\prime}$ is $\rho$-irreducible. In this case we shall say that $E^{\prime}$ itself is $\rho$-irreducible. Every pseudo-inverse semigroup with biordered set $E$ is an $\mathscr{H}$-coextension of some full subsemigroup of $T(E)$ and this is a coextension by rectangular bands of some full subsemigroup of $T\left(E^{\prime}\right)$. Thus every pseudo-inverse semigroup $S$ with $E(S)=E$ is a coextension of a full subsemigroup of $T\left(E^{\prime}\right)$ by completely simple semigroups. This is also true of any locally orthodox semigroup that is a coextension of $S$ by rectangular bands. Therefore we have

COROLLARY 2.5. Every locally orthodox semigroup is a coextension by completely simple semigroups of a fundamental pseudo-inverse semigroup whose psuedosemilattice is $\rho$-irreducible.

We now proceed to characterize those pseudo-semilattices $E$ with the property that there exists some $\rho$-irreducible pseudo-inverse semigroup $S$ with $E(S)=E$.

Let $E$ be a biordered set and let $U(E)$ denote the universal idempotent-generated semigroup of $E$ (see Nambooripad (1979)). Then $\Gamma_{U(E)}=\bar{\Gamma}_{0}$ (see Nambooripad (1979), Section 6). Since $E$ is a pseudo-semilattice, $\Gamma_{0}$ consists of identity cycles only, so $\Gamma_{0}=\bar{\Gamma}_{0}=\Gamma_{U(E)}$. Now, by Corollary 1.12, a pseudo-inverse semigroup $S$ is $\rho$ irreducible if and only if $I(S)$ has this property. Then by Corollary 2.14 of Meakin and Nambooripad (1979a), $U(E)$ is $\rho$-irreducible.

Now if $U(E)$ is $\rho$-irreducible, $E$ does not contain two elements $e, f$ such that $e \mathscr{L} f$ [or $e \mathscr{R} f]$ and

$$
\omega^{r}(e)=\omega(e), \omega^{r}(f)=\omega(f)\left[\omega^{l}(e)=\omega(e), \omega^{l}(f)=\omega(f)\right] .
$$

For, in that case, $(e, f) \in \rho(U(E))$. On the other hand, if $e U(E) \approx f U(E)(e \mathscr{L} f)$ and if $g, h \in \omega^{r}(e)$ with $g \mathscr{R} h$ then

$$
\left(\begin{array}{cc}
g & h \\
\theta(g) & \theta(h)
\end{array}\right)
$$

is a $2 \times 2$ rectangular band in $U(E)\left(\theta\right.$ is the $\mathscr{L}$-class preserving isomorphism of $\omega^{r}(e)$ onto $\omega^{r}(f)$ of Theorem 2.2). This implies that

$$
\left(\begin{array}{cc}
g & h \\
\theta(g) & \theta(h)
\end{array}\right) \in \Gamma_{\boldsymbol{U}(\boldsymbol{E})}=\Gamma_{0}
$$

so that $g=h$. Hence $\omega^{r}(e)=\omega(e)$. Similarly $\omega^{r}(f)=\omega(f)$. Thus we have the following :

THeOREM 2.6. Let $E$ be a pseudo-semillatice. Then there exists some $\rho$-irreducible pseudo-inverse semigroup $S$ with $E(S)=E$ if and only if $E$ satisfies the following condition and its dual:

(*) Let $e, f \in E$ with e $\mathscr{L} f$. If $\omega^{r}(e)=\omega(e)$ and $\omega^{r}(f)=\omega(f)$ then $e=f$. 
Let us denote the equivalence relations $\pi(S) \cap \mathscr{L}$ and $\pi(S) \cap \mathscr{R}$ on a regular semigroup $S$ by $\pi_{t}$ and $\pi_{r}$ respectively. If $\alpha$ and $\beta$ are the maximum congruences contained in $\pi_{l}$ and $\pi_{\mathrm{r}}$ respectively, then Nambooripad and Sitaraman (1979) have shown that they are the kernels of the representations introduced by Lallement (1967) and Preston (1958) (see Clifford and Preston (1961), Section 3.6). We have the following result linking these congruences to $\rho$.

THEOREM 2.7. If $S$ is a pseudo-inverse semigroup, then $\rho(S)=\alpha \circ \beta$.

ProOf. From Lemma 2.1 of Meakin and Nambooripad (1979a) it follows easily that $\rho=\left(\rho \cap \pi_{l}\right) \circ\left(\rho \cap \pi_{r}\right)$. Since $\alpha \subseteq \rho \cap \pi_{l}, \beta \subseteq \rho \cap \pi_{r}$ it is sufficient to show that $\rho \cap \pi_{l}$ and $\rho \cap \pi_{r}$ are congruences. Let $(x, y) \in \rho \cap \pi_{l}$. Then there exist $e \in E\left(R_{x}\right)$ and $f \in E\left(R_{y}\right)$ with $e \mathscr{L} f$ and $y=f x$. Let $c \in S$. If $g \in E\left(L_{\mathrm{c}}\right)$ and $k \in S(g, e)$ then we have $f k \in S(g, f)$ and $f k f=f k e$. Hence $f k f \mathscr{L} k$ and $g k=g(f k)$. So, $e f=c(f k)(f k f)=(c k)(k e)=c e$. Therefore $c y=c x$ and so $(c y, c x) \in \rho \cap \pi_{l}$. If $g^{\prime} \in E\left(R_{c}\right), h \in E\left(L_{x}\right)$ and if $k^{\prime}=h \wedge g^{\prime}$, then $x k^{\prime} \mathscr{L} y k^{\prime}$ and so

$$
x c=\left(x k^{\prime}\right)\left(k^{\prime} c\right) \mathscr{L}\left(h k^{\prime}\right)\left(k^{\prime} c\right)=y c .
$$

Therefore $(x c, y c) \in \rho \cap \pi_{l}$ and so $\rho \cap \pi_{l}$ is a congruence. Dually $\rho \cap \pi_{\mathbf{r}}$ is also a congruence.

Let $S_{1}=S / \alpha, S_{2}=S / \beta$. Then the foregoing result implies that we have the following commutative diagram :

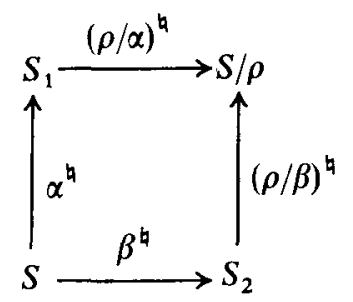

Note that by Proposition 2.12 of Meakin and Nambooripad (1979a), $\rho / \alpha=\rho(S / \alpha)=\rho\left(S_{1}\right)$ and $\rho / \beta=\rho\left(S_{2}\right)$. It is easy to check that the mapping $x \rightarrow\left(x \alpha^{\natural}, x \beta^{\natural}\right)$ is an isomorphism of $S$ onto the spined product of $S_{1}$ and $S_{2}$ relative to the homomorphisms $(\rho / \alpha)^{\natural}$ and $(\rho / \beta)$. Also, $S_{1}$ is a coextension of $S / \rho$ by left-zero semigroups and $S_{2}$ is a coextension of $S / \rho$ by right-zero semigroups. Thus

COROLlaRY 2.8. Every pseudo-inverse semigroup $S$ is a spined product of two pseudo-inverse semigroups $S_{1}$ and $S_{2}$ where $S_{1}$ is a coextension of a $\rho$-irreducible pseudo-inverse semigroup $S^{\prime}$ by left-zero semigroups and $S_{2}$ is a coextension of $S^{\prime}$ by right-zero semigroups. 
The corollary shows in particular that normal bands are spined products of left normal bands and right normal bands. This was first noted by Yamada and Kimura (1958).

\author{
Department of Mathematics \\ Department of Mathematics \\ and Statistics \\ University of Nebraska \\ University of Kerala
}

Lincoln, NE 68588, U.S.A.

\title{
References
}

K. Byleen (1977), The structure of regular and inverse semigroups (Ph.D. Thesis, University of Nebraska).

K. Byleen, J. Meakin and F. Pastijn (1980), 'The double four-spiral semigroup', Simon Stevin (to appear).

A. H. Clifford and G. B. Preston (1961), The algebraic theory of semigroups, Vol. I (Math. Surveys No. 7, Amer. Math. Soc.).

T. E. Hall (1971), 'Orthodox semigroups', Pacific J. Math. 39, 677-686.

J. M. Howie (1976), An introduction to semigroup theory (Academic Press Inc.).

G. Lallement (1967), 'Demi-groupes régulieres', Ann. Math. Pura Appl. 77, 47-129.

J. Meakin and K. S. S. Nambooripad (1979a), 'Coextension of regular semigroups by rectangular bands I', preprint, University of Nebraska.

J. Meakin and K. S. S. Nambooripad (1979b), 'Coextensions of regular semigroups by rectangular bands II', preprint, University of Nebraska.

K. S. S. Nambooripad (1979), Structure of regular semigroups I (Mem. Amer. Math. Soc. 224, Amer. Math. Soc., Providence, R.I.).

K. S. S. Nambooripad (1978), 'Pseudo-semilattices and biordered sets', preprint, University of Kerala.

K. S. S. Nambooripad and Y. Sitaraman (1979), 'On some congruences on regular semigroups', J. Algebra (to appear).

G. B. Preston (1958), 'Matrix representations of semigroups', Quart. J. Math Oxford Ser. 9, 169-176.

B.M. Schein (1972), 'Pseudo-semilattices and pseudo-lattices', Izv. Viss. Ucebn. Zaved. Matematika 2 (117), 81 -94 (in Russian).

M. Yamada and Y. Kimura (1958), 'Note on idempotent semigroups II'. Proc. Japan Acad. 34, 110-112. 